\title{
Design of the MFCS in Cutting Systems with Time-Delay
}

\author{
Dazhong Wang ${ }^{\mathrm{a}}$ and Shujing Wu \\ Shanghai University of Engineering Science, 333 Longteng Road, Shanghai 201620, China \\ awdzh168@hotmail.com
}

Keywords: cutting systems, disturbances, time-delay.

\begin{abstract}
From the last ten years, control and analysis of systems with delays is subject to lots of studies and contributions. This paper presents about the design of cutting system for time-delay. The design of the control system is constructed using the easy algebraic algorithm of matrices whose elements are polynomials of the two kinds of operators. Bounded of the inner states for the control system is given and the utility of this control design is guaranteed. It is confirmed on basis of the example that the output signal of the control system asymptotically follows the reference model signal in the case of the existence.
\end{abstract}

\section{Introduction}

The time-delay is always existent in the practical systems. Analysis of the delay phenomenon in a continuous-time domain is sophisticated [1]. There are several reasons which motivate the continuous interest and development in the time-delay systems field research. Time delays naturally appear in many physical systems [2]. Cutting process is one of the most popular manufacturing methods; however, it generates self-excited vibrations of negative effects. Specifically, they can reduce the volumetric efficiency, increase the tool wear, and decrease the geometric accuracy and surface finish [3]. Time-delay in the cutting process may result in shortened tool life and poor surface finishes, hence it should be avoided in practice. In order to predict the cutting process stability, much effort has been devoted to modeling the dynamic cutting process [4-5].

In this paper, design of model following control system (MFCS) for cutting systems with time-delay is discussed. It was proposed by [6-8]. The method of linear MFCS will be extended to cutting control system with time delays. In this case, the effectiveness of this method has verified by a numerical example and simulations. The paper structure is as follows. First, the cutting systems with time-delay are described in Section 2. The design of the cutting systems with time-delay is detailed in Section 3, an example to show the effectiveness of the proposed method in Section 4. The conclusion is finally drawn in Section 5

\section{The cutting systems with time-delay [9]}

Turing operation, as shown in Fig. 1, is investigated in this paper. With the assumption of rigid workpiece, the corresponding equation of motion is

$m \ddot{x}+c \dot{x}+k x=\cos (\beta) K_{s} b\left(h_{m}+x(t-\tau)-x(t)\right)$

where

$R_{\text {ss }}$ is spindle speed in $\mathrm{r} / \mathrm{min}$;

$\tau=60 / R_{\text {ss }}$ is the time delay;

$m$ is the modal mass;

$c$ is the modal damping;

$k$ is he modal stiffness;

$K_{s}$ is an empirical coefficient which depends on workpiece material;

$b$ is chip width; 
$\beta$ represents the force angle between $F$ and $F_{n}$;

$h_{m}$ is a constant and can be ignored on the effect of the cutting vibration.

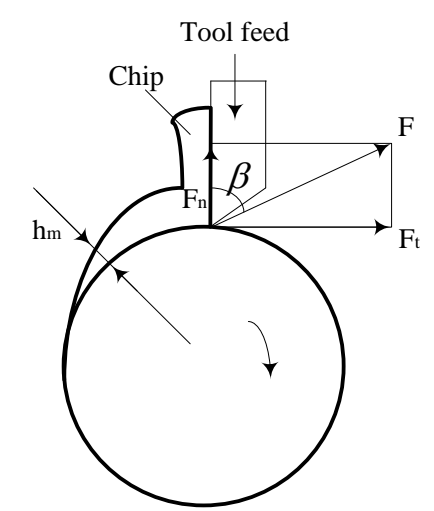

Fig. 1 Turning operation ${ }^{[9]}$

Thus, standard discrete form of (8) without time delay can be obtained as[9] $x(k+1)=A x(k)+B u(k)+d(k)$

$y(k)=C x(k)$

where $\bar{F}=\left[\begin{array}{lll}F_{0} & F_{1} & F_{2}\end{array}\right]$ and

$$
F_{0}=\left[\begin{array}{c}
F \\
0 \\
\vdots \\
0 \\
1 \\
1 \\
0 \\
0 \\
\vdots \\
0
\end{array}\right], \quad F_{1}=\left[\begin{array}{cccc}
G_{1} & 0 & \cdots & 0 \\
0 & 1 & \cdots & 0 \\
\vdots & \vdots & \ddots & \vdots \\
0 & 0 & \cdots & 1 \\
0 & 0 & \cdots & 0 \\
\vdots & \vdots & \ddots & \vdots \\
0 & 0 & \cdots & 0
\end{array}\right], \quad F_{2}=\left[\begin{array}{cccc}
G_{2} & 0 & \cdots & 0 \\
0 & 0 & \cdots & 0 \\
\vdots & \vdots & \cdots & \vdots \\
0 & 0 & \cdots & 0 \\
0 & 1 & \cdots & 0 \\
\vdots & \vdots & \ddots & \vdots \\
0 & 0 & \cdots & 1 \\
0 & 0 & \cdots & 0 \\
\vdots & \vdots & \ddots & \vdots \\
0 & 0 & \cdots & 0
\end{array}\right], \bar{G}=\left[\begin{array}{c}
0 \\
0 \\
\vdots \\
0 \\
1
\end{array}\right]
$$

Here $A \in R^{n \times n}, B \in R^{n \times m}, C \in R^{m \times n}$.

\section{Design of cutting system with time-delay}

Control law $u(t)$ can be written

$$
\begin{aligned}
& u(k)=-H x(k)+K x(k) \\
& x(k+1)=x(k)+[r-y(k)]
\end{aligned}
$$

Then we take

$$
\begin{aligned}
& {\left[\begin{array}{c}
x(k+1) \\
z(k+1)
\end{array}\right]=\left[\begin{array}{cc}
A & 0 \\
-C & I_{m}
\end{array}\right]\left[\begin{array}{c}
x(k) \\
z(k)
\end{array}\right]+\left[\begin{array}{l}
B \\
0
\end{array}\right] u(k)+\left[\begin{array}{l}
d \\
r
\end{array}\right]} \\
& y(k)=\left[\begin{array}{ll}
C & 0
\end{array}\right]\left[\begin{array}{ll}
x(k)^{T} & z(k)^{T}
\end{array}\right]^{T}
\end{aligned}
$$

Therefore, $u(k+1)$ defined by

$$
\begin{aligned}
& u(k+1)=-H x(k+1)+k Z(K+1) \\
& \quad=-\left[H\left(A-I_{m}\right)+K C\right] x(k)+\left[I_{m}-H B\right] u(k)+K r-H d
\end{aligned}
$$




\section{Example}

To illustrate some of the results presented in section 4, we consider the following example with time-delay $h_{m}=0.2$. The system is described by

$x(k+1)=2 x(k)+u(k)$

$y(k)=x(k)$

Then we have

$$
\begin{aligned}
{\left[\begin{array}{c}
x(k+1) \\
z(k+1)
\end{array}\right] } & =\left[\begin{array}{cc}
2 & 0 \\
-1 & 1
\end{array}\right]\left[\begin{array}{l}
x(k) \\
z(k)
\end{array}\right]+\left[\begin{array}{l}
1 \\
0
\end{array}\right] u(k)+\left[\begin{array}{l}
0 \\
r
\end{array}\right] \\
u(k) & =-(H,-K)\left[\begin{array}{ll}
x(k) & z(k)
\end{array}\right]^{T}
\end{aligned}
$$

and

$\left[\begin{array}{cc}2 & 0 \\ -1 & 1\end{array}\right]-\left[\begin{array}{l}1 \\ 0\end{array}\right](H,-K)=\left[\begin{array}{cc}2-H & K \\ -1 & 1\end{array}\right]$

Intrinsic value is described as follows

$\left[\begin{array}{cc}z-2+H & -K \\ 1 & z-1\end{array}\right]=z^{2}$

We have

$H=3, K=1$

Closed-loop system is given as follows

$\left[\begin{array}{c}x(k+1) \\ z(k+1)\end{array}\right]=\left[\begin{array}{ll}-1 & 1 \\ -1 & 1\end{array}\right]\left[\begin{array}{c}x(k) \\ z(k)\end{array}\right]+\left[\begin{array}{l}0 \\ r\end{array}\right]$

$y(k)=x(k)$

Let $z(0)=0$,using $x(0)=0$ and $r=1$, we have

$\left[\begin{array}{l}x(0) \\ z(0)\end{array}\right]=\left[\begin{array}{l}0 \\ 0\end{array}\right],\left[\begin{array}{l}x(1) \\ z(1)\end{array}\right]=\left[\begin{array}{l}0 \\ 1\end{array}\right],\left[\begin{array}{l}x(i) \\ z(i)\end{array}\right]=\left[\begin{array}{l}1 \\ 2\end{array}\right]$

The output responses for the above case are shown in Fig. 2. From the results, it can be seen that the control systems are valid.

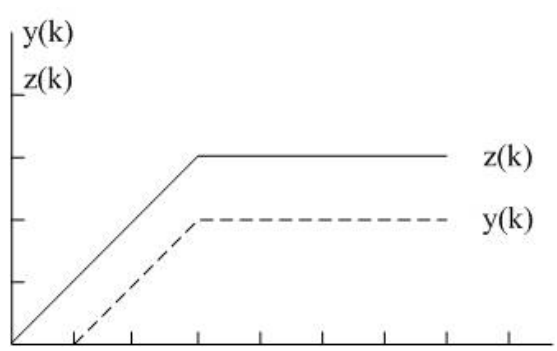

Fig. 2 Responses for the system

\section{Conclusion}

The paper discussed study of the cutting systems with time-delay. The cutting controller and corresponding control laws are designed. Based on MFCS theory, we prove the resulting closed-loop error system is uniformly ultimately bounded stable. An example is given to verify the feasibility and validity of the main results.

\section{References}

[1] Y. Zhang, K. Chong, Time-discretization of nonlinear control systems with state-delay via Taylor-Lie series, Nonlinear Analysis Vol. 70 (2009) p. 83-98. 
[2] V. Kolmanovskii, A. Myshkis, Applied theory of functional differential equations. Netherlands: Kluwer Academic Publishers 1992. P. 234.

[3] R. Rusinek, M. Wiercigroch, P. Wahi, Modelling of frictional chatter in metal cutting, International Journal of Mechanical Sciences Vol. 89 (2014) p. 167-176.

[4] K. Mao, M. Zhu, W. Xiao, B. Li, A method of using turning process excitation to determine dynamic cutting coefficients, International Journal of Machine Tools \& Manufacture Vol. 87 (2014) p. 49-60.

[5] Z. Fu, X. Zhang, X. Wang, W. Yang, Analytical modeling of chatter vibration in orthogonal cutting using a predictive force model, International Journal of Mechanical Sciences Vol. 88 (2014) p. 145-153.

[6] S. Okubo, A design of nonlinear model following control system with disturbances, Transactions of the society of Instrument and Control Engineers (Japan) Vol. 21(1985) p. 792-799.

[7] S. Wu, S. Okubo, and D. Wang, Design of model following control system for nonlinear descriptor system in discrete time, Kybernetika Vol. 44 (2008) p. 546-556.

[8] D. Wang and S. Okubo, A Design of model following control system for linear neutral system with time delays, The Transactions of The Institute of Electrical Engineers of Japan Vol. 128 (2008) p. 1657-1663.

[9] P. Zheng and X. Long, Active control of time-delay in cutting vibration, Theoretical \& Applied Mechanics Letters Vol. 3, 063003 (2013)

[10] J. Yu, C. Hu, H. Jiang, Z. Teng, Stabilization of nonlinear systems with time-varying delays via impulsive control, Neurocomputing Vol. 125 (2014) p. 68-71.

[11] H. Li, Y. Shi, Distributed model predictive control of constrained nonlinear systems with communication delays, Systems \& Control Letters Vol. 62 (2013) p. 819-826. 